\title{
Swarming Behavior as Nash Equilibrium
}

\author{
A. Bülent Özgüler* Aykut Yıldız* \\ * Bilkent University, Ankara 06800 Turkey \\ (e-mail: ozguler@ee.bilkent.edu.tr,ayildiz@ee.bilkent.edu.tr).
}

\begin{abstract}
The question of whether swarms can form as a result of a non-cooperative game played by individuals is shown here to have an affirmative answer. A dynamic game played by $\mathrm{N}$ agents in one-dimensional motion is introduced and models, for instance, a foraging ant colony. Each agent controls its velocity to minimize its total work done in a finite time interval. The game is shown to have a Nash equilibrium that has all the features of a swarm behavior.
\end{abstract}

Keywords: swarm, swarming behavior, foraging, game theory, dynamic game, Nash equilibrium.

\section{INTRODUCTION}

Swarm modeling has many application areas ranging from biological modeling (Gazi (2003)) to optimization (Bratton (2007)) and locomotion design for autonomous systems (Desai et al (1998)). In this paper, a game theoretical model is introduced to examine how swarms form as in, for instance, the foraging behavior of ant colonies or in platooning of vehicles on automated highways. This is an individual focused study of swarms that questions whether a swarm can form in a time interval by non-cooperative actions of a finite number of individuals or agents.

Game theory, in particular evolutionary game theory, has been extensively used in analyzing swarm behavior and animal decision making, (Dugatkin (1998), Giraldeau (2000) and Andrews et al (2007)). The use of game theory in social foraging, such as in Giraldeau (2000), is limited to two person games since the objective is to predict and explain the foraging behaviors of animals while in groups. Here, we assume that each agent in a group, while in search of, say, food minimizes its total effort by using the force it applies as a control input. This leads to an $N$-person infinite-dimensional dynamic game, (Basar (1999)), and to the question of whether this game has a Nash equilibrium that carries the features of a swarm. An affirmative answer means that non-cooperative optimization by $N$ individuals results in a collective behavior, namely swarming behavior. The swarm behavior is a cluster formed by the aggregation of animals of same species that move towards a target location, for instance, food source. (Vicsek (2010)) The swarm behavior is modeled here as a noncooperative distributed optimization realized by each individual. The aggregation is achieved by attraction and repulsion between individuals such that they move close to each other without collision.

The answer to whether this kind of swarming occurs due to Nash equilibrium turns out to be affirmative for particular individual cost functionals into which artificial potential energy (Gazi (2003), Gazi (2004)) terms that represents the trade-off between repulsion and attraction is incorporated.

\section{MAIN RESULTS}

A dynamic, infinite-dimensional game played by $N$ agents in one-dimensional motion is introduced in this section. A Nash equilibrium of the game is shown to exist for every specified initial positions for the agents. This equilibrium displays many known characteristics of a swarm behavior. Explicit expressions are derived for the swarm size and the distance of the swarm to the foraging location.

\subsection{Problem Definition}

One dimensional swarm behavior of $\mathrm{N}$ agents, such as the flocking of ants in a queue, will be modeled as a non-cooperative, infinite-dimensional dynamic game. It is assumed that each agent minimizes its individual total effort in a time interval by controlling its velocity. Using velocity as a control input $u^{i}(t)$ arises from applying force in a viscous environment at which particle mass is neglected (Gazi (2004)). The total work done in a finite interval $[0, T]$ that is minimized by agent- $i$ is given by

$$
\begin{aligned}
& L^{i}\left(u^{1}, \ldots, u^{N}\right):=\frac{1}{2}\left[x^{i}(T)\right]^{2} \\
& +\int_{0}^{T}\left[\sum_{j=1, j \neq i}^{N}\left(\frac{\left[x^{i}(t)-x^{j}(t)\right]^{2}}{2}-\left|x^{i}(t)-x^{j}(t)\right|\right)+\frac{\left[u^{i}(t)\right]^{2}}{2}\right] d t .
\end{aligned}
$$

Here, the first term penalizes the distance to the foraging location at the final time and serves as a very simple "attractant/repellent profile," (Gazi (2004)). The T parameter is the duration for foraging of the colony, $x^{i}(t)$ is the position of $i^{t h}$ particle at time $t$ and $N$ is the number of agents. The first term in the integrand gives the attraction potential energy, and the second term, the repulsion potential energy. These terms are introduced as a result of the assumption that each agent measures its distance to every other agent and optimizes these distances so as to remain as close as possible to every other agent without getting too close to any one of them. Introduction of such terms into the total potential energy and its (cooperative) minimization have been shown to lead to stable swarms in 
the stability analysis of Gazi (2004). The last term of the integrand in (1) is the contribution to the total work done by agent's kinetic energy. Thus, each agent minimizes its total effort, total work done, during the foraging process.

The dynamic non-cooperative game played by $\mathrm{N}$ agents is

$$
\min _{u^{i}}\left\{L_{i}\right\} \text { subject to } \dot{x}^{i}=u^{i}, \forall i=1, \ldots, N \text {. }
$$

Other more general and perhaps more realistic cost functionals are not considered here in order to keep the exposition as simple as possible.

The problem that faces each agent is an optimal control problem and necessary conditions are obtained by Pontryagin's minimum principle (see Kirk (1970) and by Theorem 6.11 of Basar (1999)). A Nash equilibrium solution exists provided the optimal solutions of $\mathrm{N}$ agents result, when simultaneously considered, in well-defined position trajectories, (Basar (1999), Section 6.3).

\subsection{Nash Equilibrium}

The existence and the general features of a Nash equilibrium of the game (2) is the main result. A Nash equilibrium, if it exists, is shown in the Appendix to be a solution of a non-linear differential equation (7) in terms of positions of the agents. Since this differential equation does not obey any local Lipschitz condition, the existence (or uniqueness) of a solution is not evident. The result below shows that there is at least one solution.

Theorem 1. There is a Nash equilibrium in which the initial ordering among the $N$ agents in the queue is preserved during $[0, T]$. Let $d(0):=\max _{i, j}\left|x^{i}(0)-x^{j}(0)\right|$ be the distance between the first and the last agent in the queue at the initial time. The Nash solution has the following properties.

P1. The distance between any two agents $i, j$ at time $t$ is given by

$$
\begin{aligned}
x^{i}(t)-x^{j}(t)= & \frac{v_{a t t}(t, T)}{\Delta(T)}\left[x^{i}(0)-x^{j}(0)\right]+ \\
& \frac{v_{r e p}(t, T)}{\Delta(T)}\left[s^{i}(0)-s^{j}(0)\right],
\end{aligned}
$$

where

$$
\begin{aligned}
\Delta(T):= & \left(1-\frac{1}{\sqrt{N}}\right) e^{-\sqrt{N} T}+\left(1+\frac{1}{\sqrt{N}}\right) e^{\sqrt{N} T} \\
v_{a t t}(t, T):= & \left(1+\frac{1}{\sqrt{N}}\right) e^{\sqrt{N}(T-t)}+\left(1-\frac{1}{\sqrt{N}}\right) e^{\sqrt{N}(t-T)} \\
v_{r e p}(t, T):= & \frac{1}{N}\left[e^{-\sqrt{N} T}\left(1-e^{\sqrt{N} t}\right)\left(1-\frac{1}{\sqrt{N}}\right)+\right. \\
& \frac{1}{\sqrt{N}}\left(e^{-\sqrt{N} t}-e^{\sqrt{N} t}\right)+ \\
& \left.e^{\sqrt{N} T}\left(1-e^{-\sqrt{N} t}\right)\left(1+\frac{1}{\sqrt{N}}\right)\right] \\
s^{i}(0):= & \sum_{k=1, k \neq i}^{N} \operatorname{sgn}\left[x^{i}(0)-x^{k}(0)\right], i=1, \ldots, N .
\end{aligned}
$$

P2. The swarm size $d_{\max }(t):=\max _{i, j}\left|x^{i}(t)-x^{j}(t)\right|$ remains bounded in $[0, T]$ for every $T$ and as $T \rightarrow \infty$ :

$$
d_{\max }(t) \leq \frac{v_{a t t}\left(t^{*}, T\right)}{\Delta(T)} d(0)+\frac{v_{r e p}\left(t^{*}, T\right)}{\Delta(T)}(2 N-2),
$$

where

$$
\begin{aligned}
t^{*}= & \frac{1}{\sqrt{N}} \ln \sqrt{\frac{f(T)}{g(T)}} ; \\
f(T)= & N(\sqrt{N}+1) e^{\sqrt{N} T} d(0)+ \\
& {\left[1-e^{\sqrt{N} T}(1+\sqrt{N})\right](2 N-2), } \\
g(T)= & N(\sqrt{N}-1) e^{-\sqrt{N} T} d(0)+ \\
& {\left[(1-\sqrt{N}) e^{-\sqrt{N} T}-1\right](2 N-2) . }
\end{aligned}
$$

The bound is attained if and only if $0 \leq t^{*} \leq T$. Maximum swarm size is attained at 0 if $t^{*}<0$ and at $T$ if $t^{*}>T$.

P3. The swarm size and the swarm center at the final time are given by

$$
\begin{aligned}
d_{\text {max }}(T) & =\frac{1}{\Delta(T)} d(0)+\frac{e^{-\sqrt{N} T}+e^{\sqrt{N} T}-2}{N \Delta(T)}(2 N-2) \\
\bar{x}(T) & =\frac{1}{T+1} \bar{x}(0) .
\end{aligned}
$$

P4. The center of the swarm $\bar{x}(t):=\frac{x^{1}(t)+\ldots+x^{N}(t)}{N}$ monotonically approaches the origin as $t \rightarrow T$ and ends up at the origin as $T \rightarrow \infty$. Moreover, as $T \rightarrow \infty$, the distances between the consecutive agents in the queue are the same.

It follows that the non-cooperative game results in a solution that has the features of a swarm and that the foraging activity of this swarm is accomplished increasingly better given sufficient time. The initial ordering of the agents in the queue is preserved at all times in this Nash solution. A closer examination of the time $t^{*}$ reveals an additional property of the swarm. If the agents start far apart from each other at the initial time, then the attraction term becomes effective and they end up closer together at the final time. Conversely, if they start close enough together, then the repellent term is more effective and they end up more apart from each other at the final time.

Expressions of the resulting optimal control inputs (velocities) of agents are rather lengthy and are not given here. However, their plots show that these are smooth functions of time and remain within reasonable limits.

Note that in defining the game we have not specified the foraging target (food supply) location but added a simple quadratic term (attractant/repellent profile) in the cost functional, which resulted in the swarm getting progressively closer to the target. The specification of the origin as the target would mean that each agent knows the exact target location at the outset. This would define a different game and will be considered elsewhere.

\section{SIMULATION RESULTS}

The optimal trajectories for $N=3$ agents are plotted to illustrate our results concerning the swarm size and the time $t^{*}$. The initial positions of the particles are set to $0,0.2$, and 0.5 , respectively. The optimal trajectories for these initial conditions are plotted in Fig. 1, for $T=1$. In this plot, there is no change in the ordering of the agents 
as postulated. The center of the swarm migrates toward 0 which is the optimal position of the foraging target.

Fig. 2 gives the swarm size, which is the distance between first and third particles. Here, swarm size attains its maximum value between $t^{*}=0.6004 \in[0, T]$. The value computed by (4) is marked in the figure by a vertical line and it is observed that the swarm size actually attains its maximum value at that time. Moreover, the swarm size at final time is calculated as $d_{\max }(T)=0.6791$, marked in 2 by a horizontal line and it coincides with the actual swarm size at the final time.

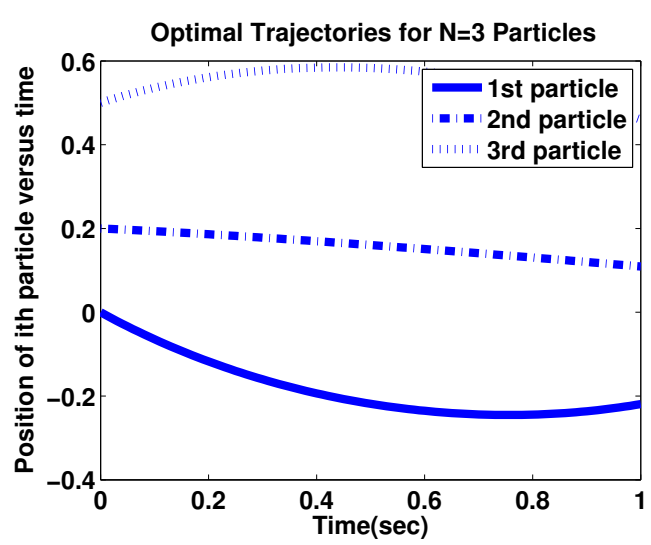

Fig. 1. Optimal trajectories for three agents.

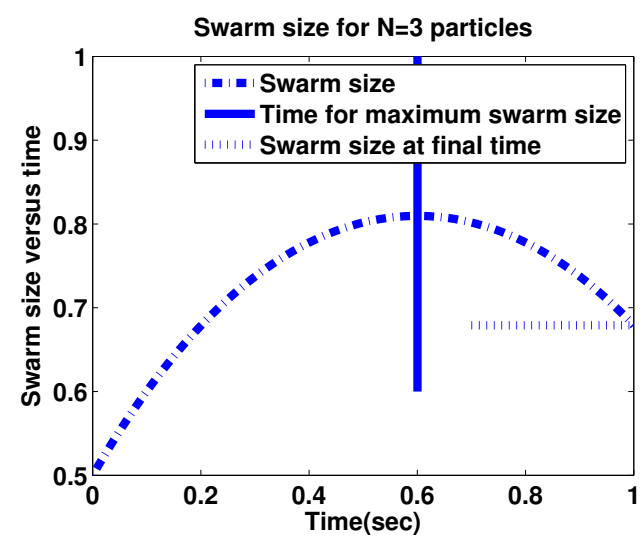

Fig. 2. Swarm size, time of maximum swarm size, and swarm size at final time.

\section{CONCLUSION}

The dynamic game model introduced in this paper can be generalized in different directions. The one-dimensional motion considered here can be extended to two and three dimensional space. Two dimensional extension would be applicable to, for instance, robot motion planning. The cost functionals used by the agents and the foraging terms in them can be made more general to cover other interesting objectives for each agent. We have assumed that every agent knows the location of all other agents. A further extension will be to relax this assumption and examine whether the game in which every other agent only knows the location of its adjacent agents has a Nash equilibrium solution. The main result of this study, that a swarm behavior can result as a (non-cooperative) Nash equilibrium of a game played by individuals, is expected to be true in all those generalizations.

\section{ACKNOWLEDGEMENTS}

The authors would like to thank the reviewers for helpful comments.

\section{REFERENCES}

Basar, T., and Olsder, G.J. (1999). Dynamic Noncooperative Game Theory. siam, Philadelphia, 1999

Dugatkin, L.A, and Reeve, H.K (1998). Game Theory and Animal Behavior. Oxford University Press, Oxford, 1998

Giraldeau, L., and Caraco, T.(2000). Social Foraging Theory. Princeton Univ. Press, Princeton, 2000

Vicsek, T., and Zafeiris, A.(2010). Collective Motion. Eprint Arxiv:1010.5017, 2010

Kirk, D.E.(1970). Optimal Control Theory. Prentice Hall, New Jersey, 1970

Gazi, V., and Passino, K.M.(2004). Stability analysis of social foraging swarms. IEEE Transactions on Systems, Man, and Cybernetics, volume 34, pages 539-557, 2004.

Gazi V., and Passino, K.M.(2003). Stability analysis of swarms. IEEE Transactions on Automatic Control, volume 48, pages 692-697, 2003.

Andrews, B.W., Passino, K.M., and Waite, T.A.(2007). Social Foraging Theory for robust multiagent system design. IEEE Transactions on Automation Science and Engineering, volume 4, pages 79-86, 2007.

Desai, J.P., Ostrowski, J., and Kumar, V.(1998). Controlling formations of multiple mobile robots Proceedings of IEEE International Conference on Robots and Automation, pages 2864-2869, 1998.

Bratton, D.(2007). Defining a standard for Particle Swarm Optimization. Swarm Intelligence Symposium, pages 120-127, 2007.

\section{APPENDIX}

The optimal control problem that faces the $i^{\text {th }}$ agent is first considered. Introducing the Lagrange multiplier $p^{i}(t)$ and minimizing the Hamiltonian

$$
H^{i}=\sum_{j=1, j \neq i}^{N}\left(\frac{\left(x^{i}-x^{j}\right)^{2}}{2}-\left|x^{i}-x^{j}\right|\right)+\frac{\left(u^{i}\right)^{2}}{2}+p^{i} u^{i}
$$

leads to the necessary conditions

$$
\begin{aligned}
& u^{i}=-p^{i}, \\
& \dot{p}^{i}=(1-N) x^{i}+\sum_{j=1, j \neq i}^{N}\left(x^{j}+\frac{x^{i}-x^{j}}{\left|x^{i}-x^{j}\right|}\right), \\
& \dot{x}^{i}=u^{i}
\end{aligned}
$$

and the boundary conditions

$$
x^{i}(0) \in \mathbf{R}, p^{i}(T)=x^{i}(T) .
$$

Let $\mathcal{I}$ denote the matrix with all entries equal to 1 . The equations (6) for all $i=1, \ldots, N$ combined can be written as

$$
\left[\begin{array}{c}
\dot{\mathbf{x}} \\
\dot{\mathbf{p}}
\end{array}\right]=\left[\begin{array}{cc}
0 & -I \\
\mathcal{I}-N I & 0
\end{array}\right]\left[\begin{array}{l}
\mathbf{x}(t) \\
\mathbf{p}(t)
\end{array}\right]+\left[\begin{array}{c}
0 \\
\mathbf{s}(t)
\end{array}\right]
$$

where

$$
\begin{aligned}
& \mathbf{x}:=\left[\begin{array}{lll}
x^{1} & \ldots & x^{N}
\end{array}\right]^{T}, \mathbf{p}:=\left[\begin{array}{lll}
p^{1} & \ldots & p^{N}
\end{array}\right]^{T}, \\
& \mathbf{s}:=\left[\sum_{j=1, j \neq 1}^{N} \operatorname{sgn}\left(x^{1}-x^{j}\right) \ldots \sum_{j=1, j \neq N}^{N} \operatorname{sgn}\left(x^{N}-x^{j}\right)\right]^{T} \text {. }
\end{aligned}
$$


Here the "signum vector" $\mathbf{s}$ is piecewise-constant in the interval $[0, T]$ with each constant value obtained by a permutation of entries in $[1-N 3-N \ldots N-3 N-1]^{T}$. This is because its $i^{\text {th }}$ entry $s^{i}=\sum_{j=1, j \neq i}^{N} \operatorname{sgn}\left(x^{i}-x^{j}\right)$ is equal to $2 B(i)+1-N$, where $B(i)$ denotes the number of agents behind the agent $i$ and can assume a value between 0 and $N-1$. Note that the vector $\mathbf{s}$ in (7) originates from the repulsion terms in the cost functionals so that the part of the solution obtained with $\mathbf{s}=0$ will be called the attraction term and summand due to $\mathbf{s}$, the repulsion term. Thus,

$$
\left[\begin{array}{c}
\mathbf{x}(t) \\
\mathbf{p}(t)
\end{array}\right]=\left[\begin{array}{l}
\mathbf{x}_{a t t}(t) \\
\mathbf{p}_{a t t}(t)
\end{array}\right]+\left[\begin{array}{l}
\mathbf{x}_{r e p}(t) \\
\mathbf{p}_{r e p}(t)
\end{array}\right]
$$

where

$$
\begin{aligned}
& {\left[\begin{array}{l}
\mathbf{x}_{a t t}(t) \\
\mathbf{p}_{\text {att }}(t)
\end{array}\right]=\left[\begin{array}{ll}
\phi_{11}(t) & \phi_{12}(t) \\
\phi_{21}(t) & \phi_{22}(t)
\end{array}\right]\left[\begin{array}{l}
\mathbf{x}(0) \\
\mathbf{p}(0)
\end{array}\right],} \\
& {\left[\begin{array}{l}
\mathbf{x}_{\text {rep }}(t) \\
\mathbf{p}_{\text {rep }}(t)
\end{array}\right]=\int_{0}^{t}\left[\begin{array}{l}
\phi_{12}(t-\tau) \\
\phi_{22}(t-\tau)
\end{array}\right] \mathbf{s}(\tau) d \tau .}
\end{aligned}
$$

Here, the partitioned matrix $\phi(t)$ is the state transition matrix of (7) when $\mathbf{s}=0$. Its partitions can be computed to be given by

$$
\begin{aligned}
& \phi_{i j}(t)=a_{i j}(t) I+b_{i j}(t)(\mathcal{I}-I) ; i, j=1,2 ; \\
& a_{11}(t)=a_{22}(t)=\frac{1}{2 N}\left[2+(N-1) e^{\sqrt{N} t}+(N-1) e^{-\sqrt{N} t}\right], \\
& b_{11}(t)=b_{22}(t)=\frac{1}{2 N}\left(2-e^{\sqrt{N} t}-e^{-\sqrt{N} t}\right), \\
& a_{12}(t)=\frac{-1}{2 N}\left(2 t+\frac{N-1}{\sqrt{N}} e^{\sqrt{N} t}-\frac{N-1}{\sqrt{N}} e^{-\sqrt{N} t}\right), \\
& b_{12}(t)=\frac{-1}{2 N}\left(2 t-\frac{1}{\sqrt{N}} e^{\sqrt{N} t}+\frac{1}{\sqrt{N}} e^{-\sqrt{N} t}\right), \\
& a_{21}(t)=\frac{1-N}{2 \sqrt{N}}\left(e^{\sqrt{N} t}-e^{-\sqrt{N} t}\right), \\
& b_{21}(t)=\frac{1}{2 \sqrt{N}}\left(e^{\sqrt{N} t}-e^{-\sqrt{N} t}\right) .
\end{aligned}
$$

Thus, each $\phi_{i j}(t)$ is a matrix with identical diagonal and identical off-diagonal entries. A solution for a given $\mathbf{x}(0) \in$ $\mathbf{R}^{N}$ of the nonlinear differential equation (7) obeying the final condition $\mathbf{x}(T)=\mathbf{p}(T)$ is a Nash equilibrium of the dynamic game (2).

Proof of Theorem 1. We first prove the existence of a Nash equilibrium. Suppose that the initial ordering among agents is preserved so that $\mathbf{s}(t)=\mathbf{s}(0)$ for all $t \in[0, T]$. Then, the repulsion term in (9) can be written as

$$
\left[\begin{array}{l}
\mathbf{x}_{r e p}(t) \\
\mathbf{p}_{\text {rep }}(t)
\end{array}\right]=\left(\int_{0}^{t}\left[\begin{array}{l}
\phi_{12}(t-\tau) \\
\phi_{22}(t-\tau)
\end{array}\right] d \tau\right) \mathbf{s}(0)=:\left[\begin{array}{l}
\psi_{1}(t, 0) \\
\psi_{2}(t, 0)
\end{array}\right] \mathbf{s}(0),
$$

where

$$
\begin{aligned}
\psi_{i}(t, 0) & =q_{i}(t) I+r_{i}(t)(\mathcal{I}-I) ; i=1,2 ; \\
q_{1}(t) & =\frac{1}{2 N}\left[\frac{1-N}{N}\left(e^{\sqrt{N} t}+e^{-\sqrt{N} t}-2\right)-t^{2}\right], \\
r_{1}(t) & =\frac{1}{2 N}\left[\frac{1}{N}\left(e^{\sqrt{N} t}+e^{-\sqrt{N} t}-2\right)-t^{2}\right], \\
q_{2}(t) & =\frac{1}{2 N}\left[2 t+\frac{N-1}{\sqrt{N}}\left(e^{\sqrt{N} t}-e^{-\sqrt{N} t}\right)\right], \\
r_{2}(t) & =\frac{1}{2 N}\left[2 t-\frac{1}{\sqrt{N}}\left(e^{\sqrt{N} t}-e^{-\sqrt{N} t}\right)\right] .
\end{aligned}
$$

Using the boundary condition $\mathbf{x}(T)=\mathbf{p}(T)$ in (8) gives

$$
\begin{aligned}
& {\left[\phi_{11}(T)-\phi_{21}(T)\right] \mathbf{x}(0)+\left[\phi_{12}(T)-\phi_{22}(T)\right] \mathbf{p}(0)+} \\
& {\left[\psi_{1}(T, 0)-\psi_{2}(T, 0)\right] \mathbf{s}(0)=0}
\end{aligned}
$$

which can be solved for $\mathbf{p}(0)$ since $\phi_{12}(T)-\phi_{22}(T)$ is nonsingular. It follows that there is a candidate solution of $(7)$ for every $\mathbf{x}(0)$. This solution is

$$
\begin{aligned}
& \mathbf{x}(t)= \\
& \left\{\phi_{11}(t)-\phi_{12}(t)\left[\phi_{12}(T)-\phi_{22}(T)\right]^{-1}\left[\phi_{11}(T)-\phi_{21}(T)\right]\right\} \mathbf{x}(0)+ \\
& \left\{\psi_{1}(t, 0)-\phi_{12}(t)\left[\phi_{12}(T)-\phi_{22}(T)\right]^{-1}\left[\psi_{1}(T, 0)-\psi_{2}(T, 0)\right]\right\} \mathbf{s}(0) .
\end{aligned}
$$

In this expression, the coefficient matrices of $\mathbf{x}(0)$ and $\mathbf{s}(0)$ inherit (from $\phi_{i j}$ and $\psi_{i}$ ) the property of having identical diagonal/off-diagonal entries, which leads to the simple expression (3) for pairwise distances. A crucial step is to verify that for any pair $i, j$

$$
\operatorname{sgn}\left[x^{i}(t)-x^{j}(t)\right]=\operatorname{sgn}\left[x^{i}(0)-x^{j}(0)\right]
$$

for all $t \in[0, T]$. To see this, we first note that $s^{i}(0)-$ $s^{j}(0)=2[B(i)-B(j)]$ so that $\operatorname{sgn}\left[s^{i}(0)-s^{j}(0)\right]=$ $\operatorname{sgn}\left[x^{i}(0)-x^{j}(0)\right]$. Next, we note that $v_{a t t}(t)>0$ and $v_{\text {rep }}(t)>0$ for all $t \in(0, T]$, where the positivity of $v_{\text {rep }}(t)$ can be shown, e.g., by examining its derivative. Finally, $\Delta(T)>0$ so that (11) holds in the whole interval. This proves that (10) is indeed a solution. The maximum swarm size at any $t \in[0, T]$ is given by

$$
\begin{aligned}
d_{\text {max }}(t)= & \frac{v_{a t t}(t, T)}{\Delta(T)} \max _{i, j}\left[x^{i}(0)-x^{j}(0)\right]+ \\
& \frac{v_{r e p}(t, T)}{\Delta(T)} \max _{i, j}\left[s^{i}(0)-s^{j}(0)\right] \\
= & \frac{v_{a t t}(t, T)}{\Delta(T)} d(0)+ \\
& \frac{v_{r e p}(t, T)}{\Delta(T)} \max _{i, j}\left[s^{i}(0)-s^{j}(0)\right],
\end{aligned}
$$

where $\max _{i, j}\left[s^{i}(0)-s^{j}(0)\right]$ is the difference between the first and last agent's signum numbers, respectively, $N-1$ and $1-N$. This yields $\max _{i, j}\left[s^{i}(0)-s^{j}(0)\right]=2 N-2$ and

$$
d_{\text {max }}(t)=\frac{v_{a t t}(t, T)}{\Delta(T)} d(0)+\frac{v_{r e p}(t, T)}{\Delta(T)}(2 N-2) .
$$

Maximizing this expression, it is easily shown that maximum is attained at $t^{*}$ of (P2) if it falls inside the interval $[0, T]$ and at the boundaries if it is outside that interval. The first expression in (P3) is obtained by evaluating (12) at $t=T$. The expression for the swarm center is obtained from (10) by taking the average of the entries of $\mathbf{x}(t)$ : 


$$
\bar{x}(t)=\left(1-\frac{t}{T+1}\right) \bar{x}(0) .
$$

Evaluating at $t=T$, the second expression in (P3) is obtained. The last property (P4) follows by (13) and by (3), where $i$ and $j$ are taken as consecutive agents in the queue, evaluating at $t=T$ and by taking the limit as $T \rightarrow \infty$.

Although, we have not resolved here whether any other Nash equilibrium exists or not, we note that the solution given in Theorem 1 is actually the unique solution of the game (2). This will be proved elsewhere. 